PROCEEDINGS OF THE

AMERICAN MATHEMATICAL SOCIETY

Volume 128, Number 1, Pages 237-243

S 0002-9939(99)05007-8

Article electronically published on May 20, 1999

\title{
AN OBSTRUCTION TO QUANTIZING COMPACT SYMPLECTIC MANIFOLDS
}

\author{
MARK J. GOTAY, JANUSZ GRABOWSKI, AND HENDRIK B. GRUNDLING \\ (Communicated by Peter Li)
}

\begin{abstract}
We prove that there are no nontrivial finite-dimensional Lie representations of certain Poisson algebras of polynomials on a compact symplectic manifold. This result is used to establish the existence of a universal obstruction to quantizing a compact symplectic manifold, regardless of the dimensionality of the representation.
\end{abstract}

\section{INTRODUCTION}

Let $(M, \omega)$ be a symplectic manifold. Consider the Poisson algebra $C_{c}^{\infty}(M)$ of all compactly supported smooth functions on $M$. In a recent paper [GM], Ginzburg and Montgomery observed:

Theorem 0. There exists no nontrivial finite-dimensional Lie representation of $C_{c}^{\infty}(M)$.

When $M$ is compact, this yields the "no-go" result:

Corollary 0. There exists no nontrivial finite-dimensional full prequantization of $M$.

(The terminology is explained in the following section.) Although not surprising on mathematical grounds, since $C_{c}^{\infty}(M)$ is "huge", this corollary does have physical import, as one expects the quantization of a compact phase space to yield a finitedimensional Hilbert space.

From the standpoint of quantization theory, however, one is more interested in certain polynomial algebras than in $C_{c}^{\infty}(M)$ or even all of $C^{\infty}(M)$. Inspired by this work, as well as that of Avez [Av1], here we generalize both Theorem 0 and Corollary 0 as follows. Let $\mathcal{B}$ be a basic set of observables on $M, \mathfrak{b}$ the Lie algebra generated by $\mathcal{B}$, and $P(\mathfrak{b})$ the Poisson algebra of polynomials generated by $\mathfrak{b}$. (See $\S 2$.) Assume that $M$ is compact and that $\mathfrak{b}$ is finite-dimensional. Our main result is:

Theorem 1. There exists no nontrivial finite-dimensional Lie representation of $P(\mathfrak{b})$.

From this we immediately obtain

Received by the editors March 11, 1998.

1991 Mathematics Subject Classification. Primary 81S99; Secondary 17 B66.

Key words and phrases. Symplectic manifolds, quantization, obstructions.

(C)1999 American Mathematical Society 
Corollary 1. There is no nontrivial finite-dimensional prequantization of $P(\mathfrak{b})$.

Furthermore, using Corollary 1, we are able to establish the following result, which holds regardless of the dimensionality of the representation.

Theorem 2. There exists no nontrivial quantization of the pair $(P(\mathfrak{b}), \mathcal{B})$.

Thus there is a universal obstruction to the quantization of a compact symplectic manifold. Theorem 2 generalizes some of the results obtained in $[\mathrm{GGH}]$ for the phase space $S^{2}$, with $\mathcal{B}=\mathrm{su}(2)$, corresponding to a spinning particle. In this instance the polynomial algebra $P(\mathrm{su}(2))$ consists of spherical harmonics. Implications of these no-go results will be discussed in the sequel.

\section{ON BASIC ALGEBRAS AND QUANTIZATION}

Suppose that $(M, \omega)$ is a connected $2 n$-dimensional symplectic manifold with corresponding Poisson bracket $\{$,$\} . We first recall from [GGT] the notion of a$ "basic set of observables" on $M$ which is central to our results.

Definition 1. A basic set of observables $\mathcal{B}$ is a finite-dimensional linear subspace of $C^{\infty}(M)$ such that: ${ }^{1}$

(B1) the Hamiltonian vector fields $X_{f}, f \in \mathcal{B}$, are complete,

(B2) $\mathcal{B}$ is "transitive", i.e. the collection $\left\{X_{f}, f \in \mathcal{B}\right\}$ spans $T M$, and

(B3) $\mathcal{B}$ is a minimal space satisfying these requirements.

Given a basic set $\mathcal{B}$, we denote by $\mathfrak{b}$ the Lie algebra generated by $\mathcal{B}$. We call $\mathfrak{b}$ a basic algebra. Throughout this paper, we assume that $\mathfrak{b}$ is finite-dimensional. We refer the reader to [On, $\S \S 1.2 .5-6]$ for the necessary background on Lie algebras, Lie groups, and their actions.

When $M$ is compact, we have the following characterization of basic algebras.

Proposition 1. Let $\mathfrak{b}$ be a finite-dimensional basic algebra on a compact symplectic manifold. Then, as a Lie algebra, $\mathfrak{b}$ is compact and semisimple. In particular, its center must be zero.

Proof. Define an inner product on $\mathfrak{b}$ according to

$$
\langle f, g\rangle=\int_{M} f g \omega^{n} .
$$

Using the identity

$$
\{f, g\} \omega^{n}=n d\left(f d g \wedge \omega^{n-1}\right)
$$

together with Stokes' Theorem, we immediately verify that

$$
\langle\{f, g\}, h\rangle+\langle g,\{f, h\}\rangle=0
$$

whence $\mathfrak{b}$ is compact. As a consequence, $\mathfrak{b}$ splits as the direct sum $\mathfrak{s} \oplus \mathfrak{z}$, where $\mathfrak{s}$ is semisimple and $\mathfrak{z}$ is the center of $\mathfrak{b}$. Furthermore, the span of any generating set of $\mathfrak{b}$ must then contain the center $\mathfrak{z}$.

Now the transitivity property (B2) implies that any function which Poisson commutes with every element of $\mathfrak{b}$ must be a constant, so that $\mathfrak{z} \subset \mathbb{R}$. But if $\mathfrak{z}=\mathbb{R}$, then, since $\mathfrak{z} \subset \mathcal{B}, \mathcal{B}$ would not be minimal, thereby violating (B3). Thus $\mathfrak{z}=\{0\}$ and $\mathfrak{b}$ is semisimple.

\footnotetext{
${ }^{1}$ Unlike in $[\mathrm{GGT}]$ we do not require that $1 \in \mathcal{B}$; this is superfluous.
} 
Although we make no use of it, we remark that the converse of Proposition 1 holds: Every finite-dimensional, compact, semisimple Lie algebra $\mathfrak{b}$ is a basic algebra on some symplectic manifold $M$. In fact, we can take $M$ to be a coadjoint orbit of maximal dimension in $\mathfrak{b}^{*}$; cf. the discussion following the proof of Theorem 4 in $\S 4$.

We denote by $P(\mathfrak{b})$ the polynomial algebra generated by $\mathfrak{b}$. Since $\mathfrak{b}$ is a Lie algebra, $P(\mathfrak{b})$ is a Poisson algebra. Note that $(i) P(\mathfrak{b})$ is not necessarily free as an associative algebra (cf. the examples in $\S 4$ ), and (ii) by definition $\mathbb{R} \subset P(\mathfrak{b})$. Let $P^{k}(\mathfrak{b})$ denote the (finite-dimensional) subspace of polynomials of minimal degree at most $k$. (Since $P(\mathfrak{b})$ is not necessarily free, the notion of "degree" is not welldefined, but that of "minimal degree" is.)

Finally, we state what it means to quantize a Lie subalgebra of observables. Again, we refer the reader to [GGT] for motivation and further discussion.

Definition 2. Let $\mathcal{O}$ be a Lie subalgebra of $C^{\infty}(M)$ containing 1. A prequantization of $\mathcal{O}$ is a Lie representation $\mathcal{Q}$ of $\mathcal{O}$ by skew-symmetric operators which preserve a common dense domain $D$ in some separable Hilbert space such that

(Q1) $\mathcal{Q}(1)=I$, and

(Q2) if the Hamiltonian vector field of $f \in \mathcal{O}$ is complete, then $\mathcal{Q}(f)$ is essentially skew-adjoint on $D$.

If $\mathcal{O}=C^{\infty}(M)$, the prequantization is said to be full.

Now suppose that $\mathcal{B} \subset \mathcal{O}$ is a basic set of observables.

Definition 3. A quantization of the pair $(\mathcal{O}, \mathcal{B})$ is a prequantization $\mathcal{Q}$ of $\mathcal{O}$ on a domain $D$ such that

(Q3) $\mathcal{Q}(\mathcal{B})=\{\mathcal{Q}(f) \mid f \in \mathcal{B}\}$ is an irreducible set, and

(Q4) $D$ contains a dense set of separately analytic vectors for $\mathcal{Q}(\mathcal{B})$.

When $\mathfrak{b}$ is finite-dimensional, (Q4) guarantees that the representation of $\mathfrak{b}$ so obtained is integrable to a representation of the corresponding simply connected Lie group. We are interested here in the case when $\mathcal{O}=P(\mathfrak{b})$.

\section{Proofs}

We now prove Theorem 1, Corollary 1 , and Theorem 2. We begin with a purely algebraic lemma. Let $P$ be an abstract Poisson algebra, i.e. an associative commutative algebra, equipped with a Poisson bracket $\{$,$\} . The Poisson bracket is a Lie$ bracket satisfying the Leibniz rule

$$
\{f, g h\}=\{f, g\} h+g\{f, h\} .
$$

The Leibniz rule implies the identity

$$
\{f, g h\}+\{g, h f\}+\{h, f g\}=0 .
$$

Proposition 2. If $L$ is a finite-codimensional Lie ideal of an infinite-dimensional Poisson algebra $P$ with identity, then either $L$ contains the derived ideal $P^{\prime}=$ $\{P, P\}$ or there is a maximal finite-codimensional associative ideal $J$ of $P$ such that $P^{\prime} \subset J$.

Proof. Let us assume that $P^{\prime}$ is not included in $L$ and let us set $U_{L}=\{f \in P \mid$ $\{f, P\} \subset L\}$.

From (3) it follows easily that $U_{L}$ is an associative subalgebra of $P$ (cf. [Gr2, 1.6(b)]). Moreover, $U_{L}$ is finite-codimensional, since it contains $L$, and $U_{L} \neq P$, since the derived ideal $P^{\prime}$ is not included in $L$. 
Let $K$ be the largest associative ideal of $P$ included in $U_{L}$, i.e. $K=\{f \in$ $\left.P \mid f P \subset U_{L}\right\}$. As the kernel of the regular representation of $U_{L}$ in $P / U_{L}$ by associative multiplication, $K$ is finite-codimensional in $U_{L}$ (and hence in $P$ ) and $K \neq P$. Moreover, $\{P, K\} \subset L \subset U_{L}$. Since for any $f \in P$ we have

$$
f\{P, K\} \subset\{P, f K\}+\{P, f\} K \subset U_{L},
$$

we can write $\{P, K\} \subset K$.

Since $P$ has an identity, $K$ is contained in some maximal (and thus prime) finitecodimensional associative ideal $J$. Let $X_{f}$ be the Hamiltonian vector field of $f \in P$. Since

$$
J^{X_{f}}=\left\{g \in P \mid\left(X_{f}\right)^{n} g \in J \quad \text { for all } n=0,1,2, \ldots\right\}
$$

is finite-codimensional (as it contains $K$ ), we conclude from [Gr1, Lemma 4.1] that $X_{f}(J) \subset J$, i.e. $\{P, J\} \subset J$. Finally, we use [Gr1, Lemma 4.2] to get that $\{P, P\} \subset J$.

Since the basic algebra $\mathfrak{b}$ is fixed, we will henceforth abbreviate the Poisson algebra $P(\mathfrak{b})$ by $P$.

Proof of Theorem 1. Suppose that $\mathcal{Q}$ were a Lie representation of $P$ on some finitedimensional vector space. Then $L=\operatorname{ker} \mathcal{Q}$ is a finite-codimensional Lie ideal of $P$. We will show that $L$ has codimension at most 1 , whence the representation is trivial (i.e. $\mathcal{Q}$ factors through a representation of $P / L$ with $\operatorname{dim} P / L \leq 1$ ). We accomplish this in two steps, by showing that:

(a) The derived ideal $\{P, P\}$ has codimension 1 in $P$, and

(b) $L \supset\{P, P\}$.

Recall that the mean of $f \in C^{\infty}(M)$ is

$$
\bar{f}=\frac{1}{\operatorname{vol}(M)} \int_{M} f \omega^{n}
$$

Let $P_{0}$ denote the Lie ideal of all polynomials of zero mean. The decomposition $f \mapsto \bar{f}+(f-\bar{f})$ gives $P=\mathbb{R} \oplus P_{0}$. Thus, if we prove that $\{P, P\}=P_{0}$, (a) will follow.

Using (2) along with Stokes' Theorem, we immediately have that $\{P, P\} \subset P_{0}$. To show the reverse inclusion, let $\left\{b_{1}, \ldots, b_{N}\right\}$ be a basis for $\mathfrak{b}$, so that

$$
\left\{b_{i}, b_{j}\right\}=\sum_{k=1}^{N} c_{i j}^{k} b_{k}
$$

for some constants $c_{i j}^{k}$. Following Avez [Av2], define the "symplectic Laplacian"

$$
\Delta f=-\sum_{i=1}^{N}\left\{b_{i},\left\{b_{i}, f\right\}\right\} .
$$

It is clear from these two expressions and the Leibniz rule that the linear operator $\Delta$ maps $P^{k}$ into $P_{0}^{k}$. Furthermore, taking into account the transitivity assumption (B2), we can apply [Av2, Prop. 1(4)] to conclude that $\Delta f=0$ only if $f$ is constant. Thus for each $k \geq 0$, the decomposition $P^{k}=\mathbb{R} \oplus P_{0}^{k}$ implies $\Delta\left(P^{k}\right)=P_{0}^{k}$. It follows that $P_{0} \subset\{P, P\}$.

If (b) does not hold, then by Proposition 2 there must be a proper associative ideal $J$ in $P$ with $\{P, P\} \subset J$. Since $\{P, P\}=P_{0}$ has codimension $1, P_{0}=J$. This is, however, impossible, since $f^{2}$ has zero mean only if $f=0$. 
Corollary 1 is trivial, since a prequantization is simply a special type of Lie representation.

Proof of Theorem 2. We proceed by reducing to the finite-dimensional case and applying Corollary 1. Suppose that $\mathcal{Q}$ were a quantization of $(P, \mathcal{B})$ on a Hilbert space. By conditions $(\mathrm{Q} 2)$ and $(\mathrm{Q} 4), \mathcal{Q}(\mathfrak{b})$ can be exponentiated to a unitary representation of the connected, simply connected Lie group $B$ with Lie algebra $\mathfrak{b}$ (recall that $\mathfrak{b}$ is assumed finite-dimensional) which, according to (Q3), is irreducible. Since by Proposition $1 \mathfrak{b}$ is compact and semisimple, $B$ is compact. The representation space must thus be finite-dimensional, and so Corollary 1 applies.

\section{Discussion}

Theorem 2 asserts that the polynomial algebra $P(\mathfrak{b})$ generated by any finitedimensional basic algebra $\mathfrak{b}$ on a compact symplectic manifold cannot be consistently quantized. We emphasize, however, that this result need not hold if $\mathfrak{b}$ is allowed to be infinite-dimensional: there exists a full quantization of the torus [Go]. (In this regard we observe that by Theorem 3 below, there is no finite-dimensional basic algebra on the torus as it is not simply connected.) Similarly, Theorem 1 and Corollary 1 can fail when the representation space is allowed to be infinitedimensional: as is well-known, full prequantizations exist provided $\omega$ is integral. Thus Corollary 1 and Theorem 2 are the optimal no-go results for compact phase spaces.

It is of interest to determine those compact symplectic manifolds which admit basic sets of observables (or, equivalently, basic algebras). It turns out that these form a quite restricted class.

Theorem 3. If a compact, connected, symplectic manifold $M$ admits a finite-dimensional basic algebra $\mathfrak{b}$, then $M$ is a coadjoint orbit in $\mathfrak{b}^{*}$. In particular, $M$ must be simply connected.

Proof. Let $\mathfrak{b}$ be a basic algebra on $M$, and denote by $\mathfrak{g}$ the Lie algebra formed by the Hamiltonian vector fields of elements of $\mathfrak{b}$. Since $M$ is compact the vector fields in $\mathfrak{g}$ are complete, so $\mathfrak{g}$ may be integrated to a symplectic action on $M$ of some connected Lie group $G$ with Lie algebra $\mathfrak{g}$. Condition (B2) implies that this action is locally transitive, and therefore globally transitive as $M$ is connected. Thus $M$ is a homogeneous space for $G$.

By Proposition 1 the center of $\mathfrak{b}$ is trivial. It follows that $\mathfrak{g} \cong \mathfrak{b}$, whence the action of $G$ on $M$ is Hamiltonian. Again using the compactness and semisimplicity of $\mathfrak{b}$, we conclude that $G$ is compact. The Kirillov-Kostant-Souriau Coadjoint Orbit Covering Theorem [MR, Thm. 14.6.5] then implies that $M$ is a symplectic covering of a coadjoint orbit $O$ in $\mathfrak{b}^{*}$. On the other hand, the coadjoint orbits of a compact connected Lie group are simply connected [Fi, Thm. 2.3.7], so that $M$ is symplectomorphic to $O$.

As a partial converse to Theorem 3, we have

Theorem 4. Let $M$ be a nonzero coadjoint orbit in $\mathfrak{b}^{*}$, where $\mathfrak{b}$ is a compact, simple Lie algebra. Then $M$ admits $\mathfrak{b}$ as a basic algebra.

Proof. The elements of $\mathfrak{b}$, regarded as (linear) functions on $\mathfrak{b}^{*}$, form a finitedimensional space of observables on $M$ closed with respect to the Poisson bracket $\{$,$\} . Their Hamiltonian vector fields are complete due to the compactness of$ 
$M$; moreover, transitivity is automatic since $M$ is an orbit. Thus $\mathfrak{b}$ satisfies (B1) and (B2). We will show below that $\mathfrak{b}$ is minimal with respect to these properties. Given this, let $\mathcal{B}$ be a subspace of $\mathfrak{b}$ which is minimal amongst all such satisfying (B2). Then $\mathcal{B}$ is a basic set and generates $\mathfrak{b}$, for otherwise the transitive Lie algebra generated by $\mathcal{B}$ would be smaller than $\mathfrak{b}$, which contradicts the minimality of $\mathfrak{b}$. It follows that $\mathfrak{b}$ is a basic algebra.

Identifying $\mathfrak{b}$ with $\mathfrak{b}^{*}$ by means of the $A d$-invariant inner product (1), we can identify adjoint and coadjoint orbits. Thus $M=\mathcal{O}_{h}$ for some $h \in \mathfrak{b}$. With respect to this identification $\{f, g\}(k)=\langle[f, g], k\rangle$ for $f, g, k \in \mathfrak{b}$. It is easy to see that the isotropy subalgebra $\mathfrak{b}_{h}=\{f \in \mathfrak{b} \mid[f, h]=0\}=[\mathfrak{b}, h]^{\perp}$, so we have the decomposition $\mathfrak{b}=\mathfrak{b}_{h} \oplus[\mathfrak{b}, h]$ with

$$
\left[\mathfrak{b}_{h},[\mathfrak{b}, h]\right] \subset[\mathfrak{b}, h] .
$$

Suppose now that $\mathfrak{b}$ is not minimal, and let $\mathfrak{a} \subset \mathfrak{b}$ be a minimal subalgebra for the orbit $\mathcal{O}_{h}$. The isotropy subalgebra $\mathfrak{a}_{h}=\mathfrak{a} \cap \mathfrak{b}_{h} \subset \mathfrak{b}_{h}$, and a calculation then yields $\mathfrak{a}=\mathfrak{a}_{h} \oplus \hat{\mathfrak{a}}$, where $\hat{\mathfrak{a}}=\mathfrak{a} \cap[\mathfrak{b}, h]$. By transitivity, $\mathfrak{a} / \mathfrak{a}_{h} \cong \mathfrak{b} / \mathfrak{b}_{h}$, so that $\hat{\mathfrak{a}}=[\mathfrak{b}, h]$ and hence $\mathfrak{a}^{\perp} \subset \mathfrak{b}_{h}$. These together with (4) imply

$$
\left[\mathfrak{a}^{\perp}, \hat{\mathfrak{a}}\right] \subset \hat{\mathfrak{a}} \subset \mathfrak{a} .
$$

Now, in view of the invariance of the inner product, $\left[\mathfrak{a}, \mathfrak{a}^{\perp}\right] \subset \mathfrak{a}^{\perp}$. In particular,

$$
\left[\mathfrak{a}^{\perp}, \hat{\mathfrak{a}}\right] \subset \mathfrak{a}^{\perp}
$$

and, combining (5) with (6),

$$
\left[\mathfrak{a}^{\perp}, \hat{\mathfrak{a}}\right]=\{0\} .
$$

Let us denote by $\mathfrak{t}$ the ideal generated in $\mathfrak{b}_{h}$ by $\mathfrak{a}^{\perp}$ :

$$
\mathfrak{t}=\mathfrak{a}^{\perp}+\left[\mathfrak{a}^{\perp}, \mathfrak{b}_{h}\right]+\left[\left[\mathfrak{a}^{\perp}, \mathfrak{b}_{h}\right], \mathfrak{b}_{h}\right]+\cdots .
$$

The Jacobi identity together with $(7)$ gives $\left[\left[\mathfrak{a}^{\perp}, \mathfrak{b}_{h}\right], \hat{\mathfrak{a}}\right]=\left[\mathfrak{a}^{\perp},\left[\mathfrak{b}_{h}, \hat{\mathfrak{a}}\right]\right]=\{0\}$ since, according to (4), $\left[\mathfrak{b}_{h}, \hat{\mathfrak{a}}\right] \subset \hat{\mathfrak{a}}$. By induction, we have from (8) and (7) that $[\mathfrak{t}, \hat{\mathfrak{a}}]=$ $\{0\}$, and hence $\mathfrak{t}$ is an ideal in $\mathfrak{b}$. Due to simplicity, $\mathfrak{t}$ and thus $\mathfrak{a}^{\perp}$ are $\{0\}$, so that $\mathfrak{a}=\mathfrak{b}$.

When $\mathfrak{b}$ is merely semisimple this result need not hold. For instance, $S^{2}$ is a coadjoint orbit in $\mathrm{su}(2)^{*} \oplus\{0\} \subset \mathrm{su}(2)^{*} \oplus \mathrm{su}(2)^{*} \cong \mathrm{so}(4)^{*}$ with basic algebra su(2), not so(4). (More generally, if there are several simple components, say $\mathfrak{b}=\mathfrak{b}_{1} \oplus \cdots \oplus$ $\mathfrak{b}_{K}$, then there will be low-dimensional orbits for which $\mathfrak{b}$ is not basic; for instance, for $h$ a regular element in a Cartan subalgebra $\mathfrak{c}$ of $\mathfrak{b}_{1}$ we have $\mathfrak{b}_{h}=\mathfrak{c} \oplus \mathfrak{b}_{2} \oplus \cdots \oplus \mathfrak{b}_{K}$, and the corresponding orbit is $B / B_{h} \cong B_{1} / C$ with basic algebra $\mathfrak{b}_{1}$, not $\mathfrak{b}$.)

When $M$ has maximal dimension in $\mathfrak{b}^{*}$, however, $\mathfrak{b}$ will be a basic algebra on $M$. Indeed, from the proof of Theorem 4 , the ideal $\mathfrak{t}$ generated by $\mathfrak{a}^{\perp}$ lies in $\mathfrak{b}_{h}$. Now, it is a well-known result of Duflo and Vergne that the isotropy algebras of maximal coadjoint orbits are abelian (see, e.g., [MR, pp. 278-280]). Therefore $t$ is an abelian ideal in $\mathfrak{b}$ so that, by semisimplicity, again $\mathfrak{t}$ and thus $\mathfrak{a}^{\perp}$ are $\{0\}$, whence $\mathfrak{a}=\mathfrak{b}$.

Given that $M$ is a coadjoint orbit in $\mathfrak{b}^{*}$, we may identify $P(\mathfrak{b})$ with the Poisson algebra of polynomials on $\mathfrak{b}^{*}$ restricted to $M$. In particular, we can take $M=$ $S^{2} \subset \mathrm{su}(2)^{*}, \mathcal{B}$ the space of spherical harmonics of degree one $(\mathcal{B}=\mathfrak{b} \cong \mathrm{su}(2))$, and $P(\mathfrak{b})$ the space of all spherical harmonics. In $[\mathrm{GGH}]$ it was shown with some effort that there is no nontrivial quantization of the pair $(P(\mathfrak{b}), \mathcal{B})$. But this now follows 
immediately from Theorem 2. A similar analysis applies to $\mathbb{C} P^{n} \subset \operatorname{su}(n+1)^{*}$, thereby answering in the affirmative a question posed in [GGH, §3].

Finally, we remark that the map $f \mapsto \bar{f}$ always provides a trivial-but nonzeroprequantization of $C^{\infty}(M)$ on $\mathbb{C}$. When the representation space is infinite-dimensional, Avez [Av1] has shown that $f \mapsto \bar{f} I$ is the only possible prequantization of $C^{\infty}(M)$ by bounded operators.

We explore the case when $M$ is noncompact in a separate work [GG].

\section{ACKNowledgments}

We would like to thank V. Ginzburg and G. Folland for helpful discussions. M.J.G. was supported in part by NSF grant 96-23083. J.G. was supported by KBN, grant No. 2 PO3A 042 10. H.B.G. was supported by a research grant from the Australian Research Council.

\section{REFERENCES}

[Av1] Avez, A. [1974] Représentation de l'algèbre de Lie des symplectomorphismes par des opérateurs bornés. C.R. Acad. Sc. Paris Sér. A, 279, 785-787. MR 50:14843

[Av2] Avez, A. [1974-1975] Remarques sur les automorphismes infinitésimaux des variétés symplectiques compactes. Rend. Sem. Mat. Univers. Politecn. Torino, 33, 5-12. MR 53:6628

[Fi] Filippini, R.J. [1995] The symplectic geometry of the theorems of Borel-Weil and PeterWeyl. Thesis, University of California at Berkeley.

[GM] Ginzburg, V.L. \& Montgomery, R. [1997] Geometric quantization and no-go theorems. Preprint dg-ga/9703010.

[Go] Gotay, M.J. [1995] On a full quantization of the torus. In: Quantization, Coherent States and Complex Structures, Antoine, J.-P. et al., Eds. (Plenum, New York) 55-62. MR 97g:58068

[GG] Gotay, M.J. \& Grundling, H. [1997] Nonexistence of finite-dimensional quantizations of a noncompact symplectic manifold. To appear in: Differential Geometry and Its Applications 1998, Slovák, J. and Kowalski, O., Eds. (Masaryk University, Brno). Preprint dg-ga/9710024.

[GGH] Gotay, M.J., Grundling, H., \& Hurst, C.A. [1996] A Groenewold-Van Hove theorem for $S^{2}$. Trans. Am. Math. Soc. 348, 1579-1597. MR 96h:81036

[GGT] Gotay, M.J., Grundling, H., \& Tuynman, G.T. [1996] Obstruction results in quantization theory. J. Nonlinear Sci. 6, 469-498. MR 97j:58054

[Gr1] Grabowski, J. [1978] Isomorphisms and ideals of the Lie algebras of vector fields. Invent. Math. 50, 13-33. MR 80g:57036

[Gr2] Grabowski, J. [1985] The Lie structure of $C^{*}$ and Poisson algebras. Studia Math. 81, 259-270. MR 87c: 17021

[MR] Marsden, J.E. \& Ratiu, T.S. [1994] Introduction to Mechanics and Symmetry. (SpringerVerlag, New York). MR 95i:58073

[On] Onishchik, A.L. [1994] Topology of Transitive Transformation Groups. (Johann Ambrosius Barth, Leipzig). MR 95e:57058

Department of Mathematics, University of Hawait, 2565 The Mall, Honolulu, Hawait 96822

E-mail address: gotay@math.hawaii.edu

Institute of Mathematics, University of Warsaw, ul. Banacha 2, 02-097 Warsaw, POLAND

E-mail address: Janusz.Grabowski@mimuw.edu.pl

Department of Pure Mathematics, University of New South Wales, P.O. Box 1, Kensington, New South Wales, 2033 Australia

E-mail address: hendrik@maths.unsw.edu.au 